the Young's modulus of centimeter-long CNT strands, which were obtained from tensile tests, ranged from $49 \mathrm{GPa}$ to $77 \mathrm{GPa}$, or approximately five times the moduli previously reported for oriented SWNT fibers and ribbons.

In the April 4 issue of Nature, B.Q. Wei, Ajayan, and co-workers at RPI and Motorola Physical Science Research Laboratories in Tempe, Ariz., describe a technique for arranging CNTs into welldefined configurations. The researchers stimulated CVD nanotube growth by exposing a $\mathrm{Si} / \mathrm{SiO}_{2}$ substrate to a xylene/ ferrocene $\left[\mathrm{C}_{8} \mathrm{H}_{10} / \mathrm{Fe}\left(\mathrm{C}_{5} \mathrm{H}_{5}\right)_{2}\right]$ vapor mixture at $800^{\circ} \mathrm{C}$. Multiwalled nanotubes (MWNTs), aligned normal to the substrate surface, were grown in blocks of micrometer-sized, densely packed cylindrical pillars whose heights were controlled to within 1-2 $\mu \mathrm{m}$. The researchers used this technique, which can grow CNTs in multiple directions simultaneously, to realize periodic arrays of vertically and horizontally aligned CNTs grown on repeating patterns of $\mathrm{SiO}_{2}$.

New techniques for manipulating the structure of CNTs will allow for the tuning of mechanical and electrical properties of nanostructures and will help researchers realize new nanoscale applications. Long continuous strands of SWNTs may eventually serve as highly conductive microcables, and wellordered nanotube patterns may prove useful in the manufacture of integrated systems and electromechanical devices, according to the research teams.

STEFFEN K. KALDOR

\section{Beryllium Compound Refractive Lenses Focus 6.5-keV X-Rays}

$X$-ray collimation and focusing is a topic well discussed in the scientific community. Optical refractive methods have been considered ineffective for x-ray wavelengths, and efforts have focused on the utilization of Fresnel diffraction, Bragg reflection, or specular reflection for x-ray focusing and collimation. However, the use of compound refractive lenses (CRLs) has become the subject of much scientific research, as the x-ray sources have evolved to brighter and more collimated characteristics. The group of H.R. Beguiristain from Adelphi Technology and R.H. Pantell from Stanford University has demonstrated the use of largeaperture compound beryllium lenses to achieve gains of 1.5 and focal lengths of $93 \mathrm{~cm}$ at $6.5 \mathrm{keV}$. The compound refractive lens was made of 160 biconcave unit lenses, each with a radius of curvature of $1.9 \mathrm{~mm}$. The use of beryllium extends the range of operation of compound refrac- tive lenses, improving transmission, aperture size, and gain.

As reported in the May 1 issue of Optics Letters, Be lenses were constructed with a maximum thickness of $0.76 \mathrm{~mm}$ and a minimum thickness of $40 \mu \mathrm{m}$ and with a mechanical aperture (diameter) of $2.29 \mathrm{~mm}$. Measurements of lens performance were performed at Stanford Synchrotron Radiation Laboratory. The line was equipped with a double-crystal monochromator capable of delivering $x$-rays from $2.4 \mathrm{keV}$ to $30 \mathrm{keV}$ with $5 \times 10^{-4}$ resolution. The nominally expected source size for this beam is $0.44 \mathrm{~mm} \times 1.7 \mathrm{~mm}$. The x-ray beam size upstream of the CRL was reduced to $1 \mathrm{~mm} \times 1 \mathrm{~mm}$ by the use of entrance slits. The dimensions of the exit slits were adjusted to below $25 \mu \mathrm{m}$ and then translated in the $x$ and $y$ directions across the focused $x$-ray beam. The $x$-ray ionization chamber was fixed downstream from the exit slit and measured the total $\mathrm{x}$-ray power passing through it.

The vertical and horizontal scanning widths were measured at various positions along the direction of beam propagation. The beam size profile as a function of the distance along the propagation axis was plotted and the beam waist (minimum beam spot size) was determined to be $42 \mu \mathrm{m}$, full width at half maximum



Circle No. 4 on Inside Back Cover 
(FWHM), at a position $93 \mathrm{~cm}$ downstream from the CRL using 6.5-keV photons. The spot diameter was larger than expected, which was attributed to surface irregularities or the uncertainty of the source.

The Gaussian transmission profile FWHM of the Be CRL was also measured by scanning a $25 \mu \mathrm{m} \times 25 \mu \mathrm{m}$ beam across the CRL and was determined to be $321 \mu \mathrm{m}$ with on-axis transmission of $9 \%$ at $6.5 \mathrm{keV}$ photon energy. The attenuation aperture was thus calculated to be $607 \mu \mathrm{m}$. Consequently, using the experimental data, a gain of 1.5 was calculated for this lens, compared with a theoretical value of 6 . The obtainable gain is dependent on the source size used for the experiments, and much higher gains would be expected from thirdgeneration and beyond $x$-ray facilities.

According to the researchers, the use of CRLs made of beryllium "can achieve submeter focal lengths at lower x-ray energies $(\sim 6.5 \mathrm{keV})$ than for previously reported CRLs and still have what are believed to be the largest reported apertures $(\sim 600 \mu \mathrm{m})$. Thus, it is expected that Be CRLs can outperform lenses constructed of higher-atomic-number materials at energies below $30 \mathrm{keV}$."

Calin Miclaus

\section{Heating Allows Birefringence Tuning in Microstructured Optical Fibers Partially Filled with a Polymer}

Recently, researchers have shown that microstructured photonic-crystal optical fibers (MOFs) formed by incorporating air holes running along the length of the fiber can enable additional control of birefringence, dispersion, and nonlinearity of the fibers. Among other novel techniques, introducing active materials into the air holes of microstructured optical fibers (MOFs) improves the capabilities of the devices fabricated. As they demonstrate in their article in the May 15 issue of Optics Letters, C. Kerbage, B.J. Eggleton, and coworkers from OFS Fitel Laboratories (now OFS Laboratories) in New Jersey, were able to induce and tune birefringence in MOFs.

The researchers chose MOFs comprised of a central germanium-doped core, $8 \mu \mathrm{m}$ in diameter, encircled symmetrically by six air holes. They fabricated an all-fiber polarization controller, $125 \mu \mathrm{m}$ in diameter but tapered along $1 \mathrm{~cm}$ of the length down to $\sim 30 \mu \mathrm{m}$ in diameter. This was to ensure that the mode to guide light was by total internal reflection at the silica-airhole interface. The fiber was tapered adiabatically to achieve low intrinsic loss. Since MOFs with a rotational symmetry larger than twofold are not birefringent, the scientists had to break the sixfold symmetry by filling two opposite air holes with an acrylate-based polymer. The monomer filling, limited to the tapered area, was fed at $0.01 \mathrm{~cm} / \mathrm{s}$ and cured using UV rays for $15 \mathrm{~min}$. The resulting polymer had a refractive index of 1.434 at ambient temperature and at a wavelength of $1550 \mathrm{~nm}$, although it is temperature-dependent $\left(\mathrm{d} n / \mathrm{d} T \sim-4 \times 10^{-4}{ }^{\circ} \mathrm{C}^{-1}\right)$, and hence is controlled by heating. After placing the device in a capillary heater, the research team applied a 1550-nm polarized laser beam to the fiber to yield an output light beam, which was analyzed for its polarization properties and converted into the Stokes parameters. Results after heating increments were expressed as a rotation of the Stokes vector, and all results were plotted on the Poincaré sphere.

The beam propagation method (BPM) simulated the distortion in the mode fields of this structure. Calculations performed using BPM were close to the experimental results. When the index is lower than that of silica, the light is guided by total internal reflection, and birefringence is low. When the infused polymer has an index near to that of silica, the mode of reflection becomes more asymmetric, and birefringence increases. Analysis of the polarization measurements using the Poincaré sphere show three rotations $(6 \pi)$ along the heating range of the capillary heater. Also, as the researchers reported, the calculated birefringence change reached $\sim 4.4 \times 10^{-4}$, and the tuning coefficient was $\sim 15 \mathrm{rad} / \mathrm{m}^{\circ} \mathrm{C}$. Hysteresis effects were negligible.

$$
\text { SIARI S. SOSA }
$$

\section{Osmium Exhibits Lowest Experimentally Determined Compressibility}

A group of researchers at Lawrence Livermore National Laboratory have determined through experiment and theoretical calculations that the element osmium (Os) has a lower compressibility (or higher bulk modulus $B_{0}$, the reciprocal of compressibility) than diamond, the hardest and least compressible material known to date. As reported by $\mathrm{H}$. Cynn and coworkers in the April 1 issue of Physical Review Letters, the experimentally determined value of $B_{0}$ for osmium is $462 \mathrm{GPa}$, compared with $443 \mathrm{GPa}$ for diamond. Artificially quenched metastable phases of $\mathrm{ZrO}_{2}$ and $\mathrm{TiO}_{2}$ have been reported to have bulk moduli close to that of diamond at best. A simple chemical bonding model and estimates based on the cohesive energy have suggested that Os should have a large $B_{0}$. In addition, Os has the highest Vickers hardness among the $5 d$ transition metals.
Using synchrotron x-ray sources at the National Synchrotron Light Source (Brookhaven National Laboratory) and the Stanford Synchrotron Radiation Laboratory (Stanford University), diamond-anvil cell-compression studies were carried out for three transition metals: ruthenium $(\mathrm{Ru})$, iridium (Ir), and osmium. Condensed Ar was used as a pressure medium. Pressure information was determined using luminescence from ruby grains (grain size $<3 \mu \mathrm{m}$ ). Powder samples of $\mathrm{Ru}$, Ir, and Os (grain size $<5 \mu \mathrm{m}$ ) were packed into a small hole drilled into a rhenium gasket. Synchrotron x-ray diffraction measurements yielded lattice compressions.

A plot of the reduced volume as a function of pressure using the Birch-Murnaghan equation of state (EOS) fits for the three test materials, as well as for tungsten and diamond, shows that Os exhibits the least contraction as the external pressure is increased. The Holzapfel and Vinet EOS models were also applied to the experimental data to ensure consistency of the results for $B_{0}$. The three fitting algorithms all yielded identical results, in order of decreasing compressibility: Ru, Ir, diamond, Os. Theoretical estimates of $B_{0}$ were obtained using a full-potential linear muffin-tin orbital method to calculate the EOS. The calculated values of $B_{0}$ for the $5 d$ transition metals show the same trend as the experiments.

According to the research team, this result opens new possibilities in the search for superhard materials among transitionmetal compounds and alloys.

JUNE LAU

\section{Nanoparticle Mediates Improvement in $\mathrm{Si}$ Electroluminescence Efficiency}

Researchers at National Taiwan University have obtained a several-ordersof-magnitude electroluminescence (EL) efficiency increase from a metal oxide semiconductor (MOS) tunneling diode by replacing the oxide layer within the device with a layer of $\mathrm{SiO}_{2}$ nanoparticles (NPs). C.F. Lin and co-workers have determined that the improved EL is the result of an increase in the density of radiative recombination states in the diode that stems from the spatial confinement of the charge carriers in the nanoparticles. The EL efficiency improvement allows for the use of $\mathrm{Si}$ in optoelectronic devices.

As described in the May 1 issue of Optics Letters, the researchers prepared the tunneling diodes to study the improvement by first removing the oxide layer from Si wafers. This was followed by spincoating 12-nm $\mathrm{SiO}_{2} \mathrm{NPs}$ onto the wafers. $\mathrm{Al}$ was evaporated on top of the NP layer 